\title{
Optic Nerve Head Drusen, Myopia and Ocular Hypertension: A Case Report
}

\author{
Arzu Taskiran Comez ${ }^{*}$, Baris Komur \\ Department of Ophthalmology, School of Medicine, Canakkale Onsekiz Mart University, Canakkale, Turkey. \\ Email: ${ }^{*}$ arzucomez@yahoo.com
}

Received December $10^{\text {th }}, 2013$; revised January $11^{\text {th }}, 2014$; accepted January $22^{\text {nd }}, 2014$

Copyright (C 2014 Arzu Taskiran Comez, Baris Komur. This is an open access article distributed under the Creative Commons Attribution License, which permits unrestricted use, distribution, and reproduction in any medium, provided the original work is properly cited. In accordance of the Creative Commons Attribution License all Copyrights @ 2014 are reserved for SCIRP and the owner of the intellectual property Arzu Taskiran Comez, Baris Komur. All Copyright (C 2014 are guarded by law and by SCIRP as a guardian.

\section{ABSTRACT}

A 20-year-old male patient presented at our clinic complaining of strange visual experiences (neither decreased nor blurred) and a mild headache while reading. His best corrected visual acuity revealed 20/20 with a fort myopic correction. Intraocular pressures measured with Dynamic Contour Tonometer (DCT) (Swiss Microtechnology AG, Port, Switzerland) were $23.3 \mathrm{mmHg}$ in the right eye (OD) and $27.4 \mathrm{mmHg}$ in the left eye (OS) with central corneal thicknesses (CCTs) of 588 (OD) and 591 (OS) microns, respectively. Optic discs were bilateral pale with indistinct and scalloped margins without cupping. Red free photos showed autofluorescence of both optic discs, representative of optic disc drusen. Visual field analysis (VFA) of both eyes revealed sensitivity depression with localized defects consistent with the Optical Coherence Tomography (OCT) findings. Optic nerve head drusen (ONHD), myopia and high intraocular pressures (IOPs), may cause ganglion cell damage resulting in RNFL thinning and visual field loss. Because of the difficulty in detecting the exact cause and the extent of the damage, patients with ONHD associated with high IOPs and myopia should be examined closely with serial monitoring using OCT and VFA. In case of RNFL thinning and visual field defects, topical antiglaucomatous therapy should also be determined.

\section{KEYWORDS}

Optic Disc Drusen; Myopia; Glaucoma; Intraocular Pressure

\section{Introduction}

Optic nerve head drusen (ONHD) is congenital, calcified hyaline bodies of the optic nerve causing a lumpy appearance and scalloped borders of the nerve often resulting in a wrong diagnosis of papilledema [1,2]. ONHD are $75 \%$ - 85\% bilateral and are discovered incidentally during routine eye examinations with a frequency of $1 \%$ while increasing to $3.4 \%$ with a known family history [3]. There are various hypotheses for the pathogenesis of ONHD such as; small scleral canal, congenitally dysplastic disc, and altered axoplasmic transport secondary to congenitally crowded optic nerve heads [3]. ONHD is usually detected through clinical examination but can also be confirmed by B-scan ultrasonography, CT scan,

\footnotetext{
${ }^{*}$ Corresponding author.
}

autofluorescence on red-free photography, or cross-sectional OCT imaging. Although patients with ONHD are often asymptomatic, they may have symptoms such as peripheral vision abnormalities, transient visual problems (i.e., flickering or "graying out"), and progressive visual field loss similar to glaucoma [1,4]. Patients do not complain of central vision loss because central visual acuity is generally spared with optic disc drusen. Axial myopia, which is reported as a risk factor for glaucoma, is another issue with fundus changes complicating glaucoma diagnosis and management [5].

We present a case of bilateral ONHD with high intraocular pressures (IOPs), thick corneas, and high myopia. In addition, the retinal nerve fiber layer thickness measured by OCT was found to be bilaterally decreased. The work presented here highlights the importance of optical 
coherence tomography (OCT) in evaluating and monitoring the retinal nerve fiber layer (RNFL) thinning as a consequence of ONHD associated with high IOP and myopia.

\section{Case Report}

A 20-year-old male patient complained of strange visual experiences (neither decreased nor blurred for 2 years) and a mild headache while reading. His best corrected visual acuity was $20 / 20$ with $-5.25-0.50160$ correction in the right eye (OD) and 20/20 with $-5.00-0.50170$ prescription in the left eye (OS). No relative afferent pupillary defect (RAPD) was present. The patient was able to see all the Ishihara plates with both eyes. Upon cover testing, he was orthotropic at both far and near distances. IOPs were measured with DCT (Swiss Microtechnology AG, Port, Switzerland) and were determined to be 23.3 $\mathrm{mmHg}$ in the right eye and $27.4 \mathrm{mmHg}$ in the left eye with central corneal thicknesses (CCTs) of 588 (OD) and 591 (OS) microns, respectively. Axial lengths were 24.94 $\mathrm{mm}$ (OD) and $25.02 \mathrm{~mm}$ (OS), while gonioscopy deter- mined angles to be totally open to ciliary body band in both eyes. Fundoscopic examination revealed bilateral, pale optic disc heads with indistinct and scalloped margins that appeared as swollen. No venous congestion was present.

The fundus photo, red-free photo, and the 3-D OCT photo of the right optic disc were notable for multiple drusen, particularly in the superior and superonasal portion of the optic nerve with no apparent physiological cup (Figures 1(A)-(C)). RNFL thicknesses in the superior, nasal, and inferior quadrants of the right eye revealed severe thinning (84, 52, and 92 microns, respectively) consistent with drusen localizations (Figure 1(D)). The temporal quadrant thickness was 71 microns. The visual field analysis (VFA) of the right eye showed only a few small localized nonspecific defects in the superior, inferonasal, and inferior quadrants with sensitivity depression indicating ganglion cell damages at the drusen localizations, axonal damage due to high IOP, and ischemic injury due to crowding within the disc (Figure 1(E)).

The fundus, red-free, and 3-D photo of the left optic disc showed prominent optic nerve head with autofluo-
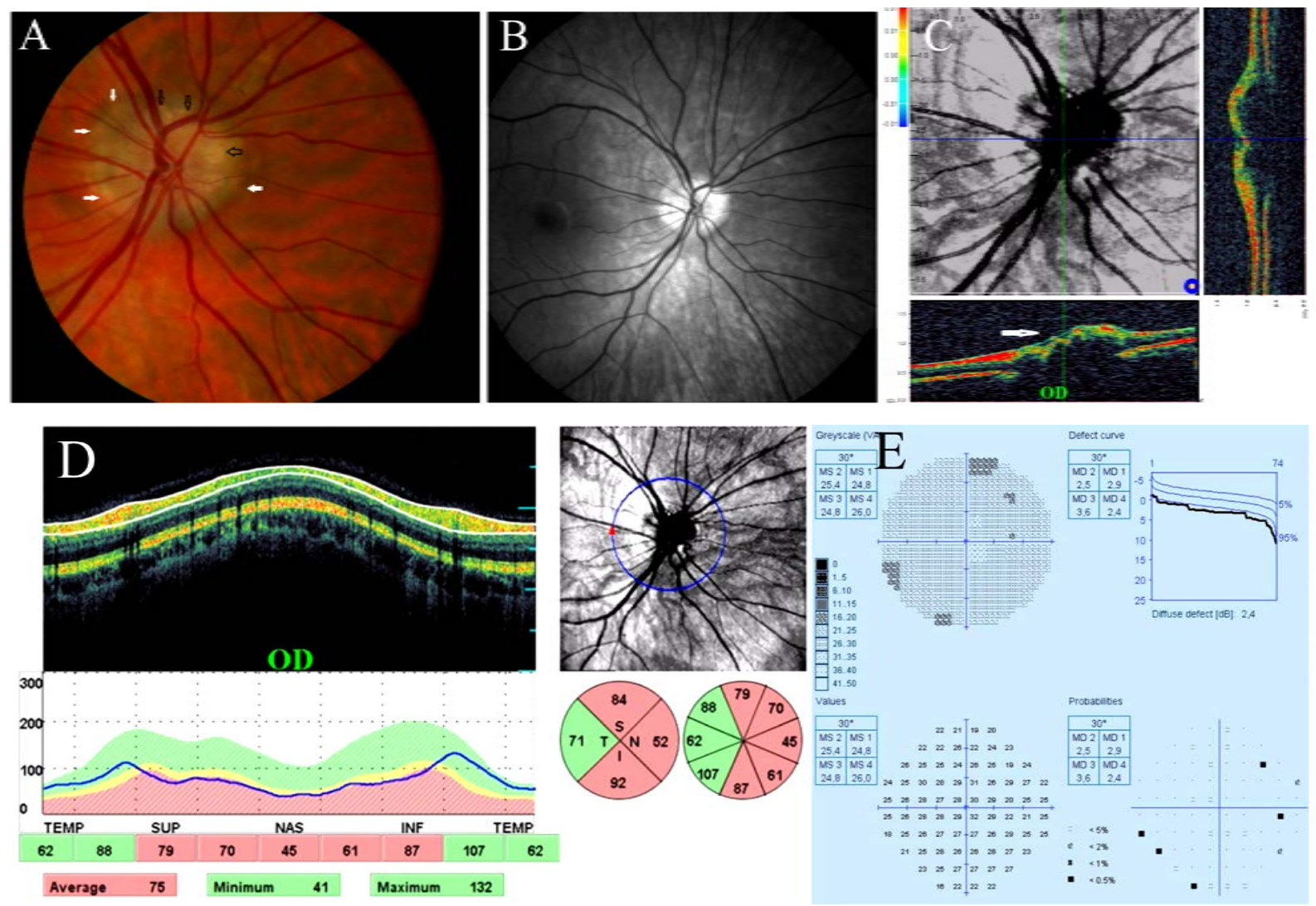

Figure 1. (A) ONHD are dominantly seen in the superior nasal border of the right optic disc (black arrows indicate areas of drusen). See the blurred borders and disappeared excavation (white arrow); (B) Yellow deposits on the disc correspond to areas of autoflourescence on the red-free photos; (C) 3-Dimentional photo of the optic disc by OCT. Prominent right optic nerve head due to drusen (arrow); (D) RNFL analysis of the right optic nerve by Spectral D-OCT. RNFL thicknesses are thin in the superior, nasal and inferior quadrants where the drusen are most abundant; (E) VFA of right eye with small localized defects and decreased sensitivity. 
rescence in the superior, nasal, and inferior quadrants (Figures 2(A)-(C)) where the RNFL thicknesses were found to be thinned (76, 57, and 79 microns, respectively), which were comparable with VFA (Figures 2(D) and (E)). The RNFL thickness of the temporal quadrant, where no visible drusen was detected, was found to be much thinner than the right eye (56 (OS) versus 71 (OD) micro- ns). The patient was ordered to return every 6 months for IOP measurements, dilated fundus examinations, VFA and RNFL analysis by OCT to monitor for progression of his condition. In the 6th month control, no difference was detected in the RNFL thickness of the right eye (Figure 3(A)). However, RNFL in the inferior and nasal quadrants of the left eye were decreased between $17.7 \%$ and $31.5 \%$, respectively compared to the initial RNFL thickness (Figure 3(B)).The VFAs were compatible with OCTs (Figures 3(C) and (D)). The IOPs were $22.4 \mathrm{mmHg}(\mathrm{OD})$ and $26.5 \mathrm{mmHg}$ (OS). Topical ocular hypotensive treatment (Alphagan 0.15\%) was initiated b.i.d. in the left eye to decrease high IOP and the patient has been ordered to return every 3 months for IOP measurements, dilated fundus examinations, VFA, and RNFL analysis by OCT to monitor for progression of his condition.

\section{Discussion}

Diagnosing optic disc drusen is clinically important because of the similar appearance of the disc as indisc edema and also the probability of overlooking co-existing glaucoma due to loss of optic disc excavation $[1,2]$. As a result of progressive drusen formation, the earliest and the most common symptoms of ONHD are abnormalities in peripheral vision or transient visual problems which may also be seen in glaucoma cases [1]. Myopia is reported as a risk factor for glaucoma, and myopic fundus changes may complicate glaucoma diagnosis and management [5]. High myopia with long axial length indicated that the globe is elongated causing a thin retina and sclera, which may cause thinning of the RNFL. OCT study results revealed that RNFL measurements were lower in the highly myopic eyes than in eyes with low to moderate myopia, and a linear correlation between RNFL thickness and axial length/spherical equivalent was present [5]. Ozdek et al. studied the relation between
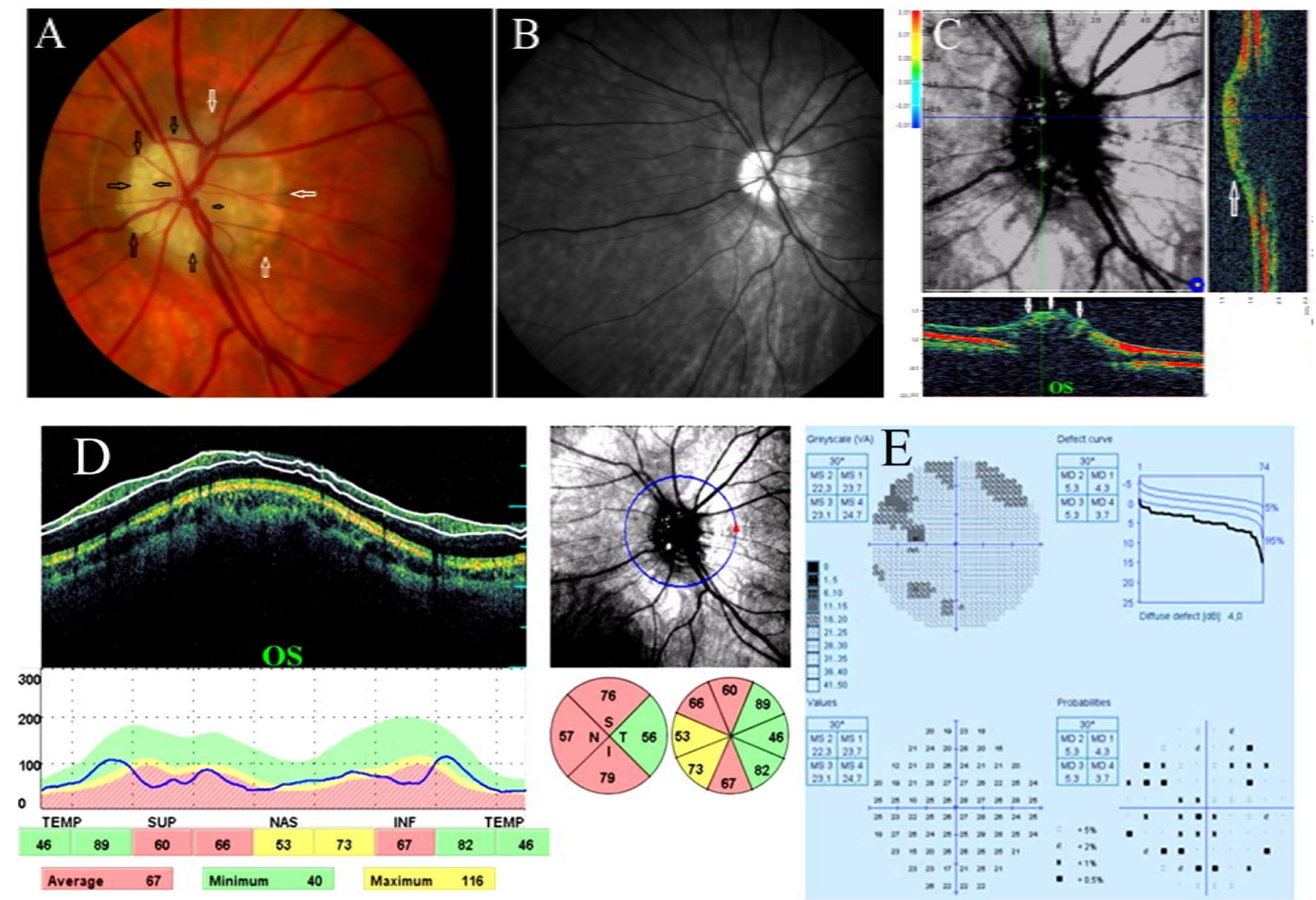

Figure 2. (A) ONHD appears nearly covering all, superior, nasal and inferior quadrants concentrically, in the left eye (OS) (black arrows). No excavation and hyperemia are evident in the left optic disc head either. Note the blurred disc margins in OS, as in OD (white arrows); (B) Red-free photo shows marked autoflourescence of the drusen localized in the superior, nasal and inferior quadrants of the left eye (OS); (C) 3-Dimensional photo of the optic disc by OCT. Prominent left optic nerve head due to drusen (arrow); (D) RNFL analysis of the left optic nerve by Spectral D-OCT. RNFL is thin in superior, nasal and inferior quadrants where the drusen are most abundant; (E) Visual field analysis (VFA) of the left eye demonstrating localized superior, superotemporal and superonasal defects, with small inferotemporal and inferior defects. 

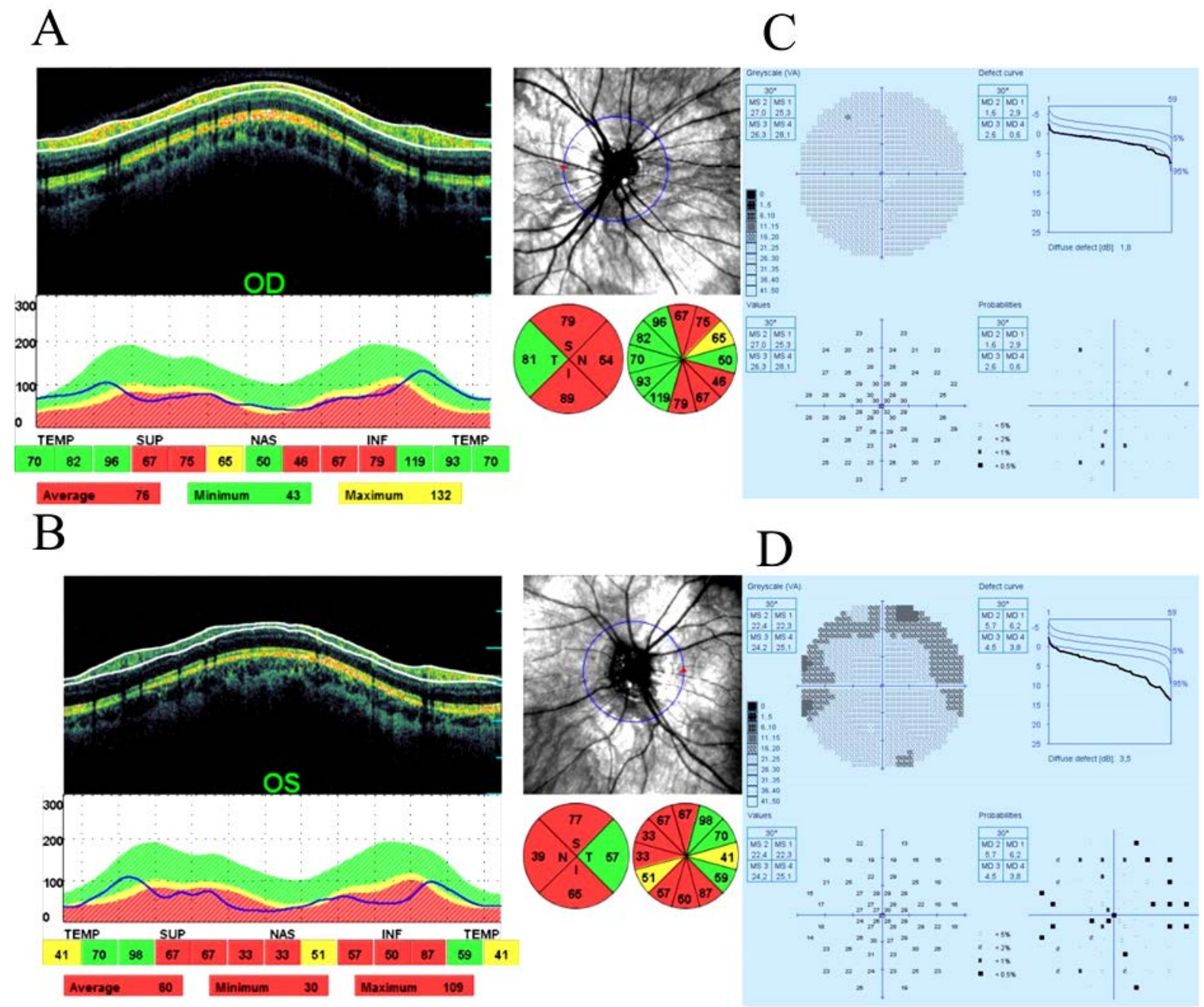

Figure 3. (A) RNFL analysis of the right optic nerve by Spectral D-OCT at the 6th month follow-up. RNFL thicknesses are thin in the superior, nasal and inferior quadrants however no progression is detected compared to initial analysis; (B) RNFL analysis of the left optic nerve by Spectral D-OCT at the 6th month follow-up. RNFL is thin in superior, nasal and inferior quadrants and progression is detected especially in the inferior and nasal quadrants; (C) VFA of right eye at the 6th month follow-up, with no defect but decreased sensitivity; (D) Visual field analysis (VFA) of the left eye at the 6th month follow-up, demonstrating concentric superior visual field defect extending from nasal to temporal quadrant.

RNFL and refractive error using the first generation of Scanning Laser Polarimetry (SLP) and showed that there was a gradual decrease in the superior and inferior RNFL with increasing myopia. Hence for each diopter decrease in spherical equivalent, there were -0.122 and -0.092 $\mu \mathrm{m}$ reductions in superior and inferior RNFL thicknesses, respectively [6]. Despite novel developments in imaging technologies, it may be quite difficult to tell whether progressive visual field loss is from ONHD, glaucoma, long axial length, or all three combined. Autofluorescence and B-scan images can detect the presence of ONHD, but they cannot show the progress or the severity of the thinning in the RNFL. In addition, they cannot determine or discriminate the cause or the causes. In our case, the drusen localizations in the right optic disc were correlated with OCT results as thinning was detected in those areas even though the VFA findings were insignificant. This result suggests a possibility of ganglion cell damage in the drusen localizations which have not manifested as a visual field defect yet. Visual field defects generally progress at a very slow rate, often without patient's awareness. It is known that in patients with glaucoma that approximately $25 \%$ to $40 \%$ of retinal ganglion cells were lost before abnormalities were statistically detected by VFA [7]. Our patient's IOP measurements by DCT were 23.3 (OD) and 27.4 (OS). DCT was designed to measure IOP independently of corneal properties. It can obtain more precise IOP measurements in glaucoma patients or ocular hypertension in cases where the measurement is debatable due to a very thin or very thick cornea [8]. Our patient's corneas were thick as determined by DCT measurements. The evidence of asym- 
metrically higher IOP and thinner temporal quadrant in the left eye made us think about other evident causes for temporal thinning such as co-existence of glaucoma as well as ONHD and axial myopia in the left eye. A study on the comparison of visual field loss in eyes with ONHD with or without ocular hypertension (OHT) found that VFL was present in $90.9 \%$ of hypertensive eyes compared with $66.7 \%$ of normotensive eyes, both with ONHD [9]. However, visual field defects with no visible drusen may also be due to axonal compression by a buried drusen located deeper in the nerve. As the optic disc in myopia is larger and sometimes tilted, VFA and OCT results may mimic glaucoma. Myopic retinal degeneration is another issue that leads to a difficulty in monitoring, as well as detecting, the cause and the extent of RNFL thinning. Spalding suggested in his report that a treatment regimen for optic nerve drusen, ocular hypertension, and visual-field defects, regardless of the etiology for the visual field defects should be designed to reduce the intraocular pressure to a level that would lower the risk of optic nerve damage [10]. Morris et al., also shares the same sentiment that ocular hypotensive agents may be used to lower intraocular pressure prophylactically to prevent further nerve fiber layer and optic nerve damage in cases with ONHD [11].

\section{Conclusion}

The current presented case demonstrates the importance of RNFL thickness measurements using OCT along with VFAs, IOP measurements, and fundus photos in patients with ONHD. The co-existence of elevated IOP and myopia may complicate the diagnosis, monitoring, and the prognosis of the optic nerve damage that may be caused by drusen. Decreasing IOP may prevent or slow down the progression of RNFL loss and decrease the damage due to probable vascular obstruction with optic nerve crowding.

\section{Conflict of Interests}

The authors declare no conflict of interest. Informed consent is provided from the patient.

\section{REFERENCES}

[1] P. Davis and W. Jay, “Optic Disc Nerve Head Drusen,”
Seminars in Ophthalmology, Vol. 18, No. 4, 2003, pp. 222-242. http://dx.doi.org/10.1080/08820530390895244

[2] M. Tso, "Pathology and Pathogenesis of Drusen of the Optic Nervehead,” Opthalmology, Vol. 88, No. 10, 1981, pp. 1066-1080. http://dx.doi.org/10.1016/S0161-6420(81)80038-3

[3] C. Auw-Haedrich, F. Staubach and H. Witschel, "Optic Disc Drusen,” Survey of Ophthalmology, Vol. 47, No. 6, 2002, pp. 515-532. http://dx.doi.org/10.1016/S0039-6257(02)00357-0

[4] J. Giovannini, "Pseudopapilledema," 2005. www.eMedicine.com

[5] C. K. Leung, S. Mohamed, K. S. Leung, C. Y. Cheung, S. L. Chan, D. K. Cheng, A. K. Lee, G. Y. Leung, S. K. Rao and D. S. Lam, "Retinal Nerve Fiber Layer Measurements in Myopia: An Optical Coherence Tomography Study," Investigative Ophthalmology \& Visual Science, Vol. 47, No. 12, 2006, pp. 5171-5176. http://dx.doi.org/10.1167/iovs.06-0545

[6] S. C. Ozdek, M. Ono, G. Gurelik and B. Hasanreisoglu, "Scanning Laser Polarimetry in Normal Subjects and Patients with Myopia,” British Journal of Ophthalmology, Vol. 84, No. 3, 2000, pp. 264-267.

http://dx.doi.org/10.1136/bjo.84.3.264

[7] H. A. Quigley, G. R. Dunkelberger and W. R. Green, "Retinal Ganglion Cell Atrophy Correlated with Automated Perimetry in Human Eyes with Glaucoma," American Journal of Ophthalmology, Vol. 107, No. 5, 1989, pp. 453-464.

[8] Y. Lachkar, "I Have Tested for You. The Contour Tonometer. IOP Analysis Using 'Dynamic Contour Tonometry',” Journal Français d'Ophtalmologiey, Vol. 29, Supplement 2, 2006, pp. 32-35. http://dx.doi.org/10.1016/S0181-5512(06)73952-3

[9] T. M. Grippo, W. A. Shihadeh, M. Schargus, E. Gramer, C. Tello, J. M. Liebmann and R. Ritch, "Optic Nerve Head Drusen and Visual Field Loss in Normotensive and Hypertensive Eyes,” Journal of Glaucoma, Vol. 12, No. 2, 2008, pp. 100-104. http://dx.doi.org/10.1097/IJG.0b013e31814b995a

[10] J. M. Spalding, "Visual-Field Loss with Optic Nerve Drusen and Ocular Hypertension: A Case Report,” Optometry, Vol. 73, No. 1, 2002, pp. 24-32.

[11] R. W. Morris, J. M. Ellerbrock, A. M. Hamp, J. T. Joy, P. Roels and C. N. Davis Jr., "Advanced Visual Field Loss Secondary to Optic Nerve Head Drusen: Case Report and Literature Review," Optometry, Vol. 80, No. 2, 2009, pp. 83-100. http://dx.doi.org/10.1016/j.optm.2008.11.004 